\title{
ESTUDIO DE \\ COMPETITIVIDAD \\ DE LAS PYMES \\ EXPORTADORAS \\ DE LA REGIÓN \\ DE VILLA MARÍA, ARGENTINA
}

\section{RESUMEN}

En el presente trabajo, se aborda la problemática de competitividad de las PyMEs exportadoras de la región de Villa María. Se realiza un comparativo con sus pares iberoamericanas, a partir de la metodología suministrada por la FAEDPYME (Fundación para el Análisis Estratégico y Desarrollo de la Pequeña y Mediana Empresa), a efectos de hacer comparables los resultados. Se busca determinar el nivel de competitividad de nuestras empresas en comparación con las del resto de Iberoamérica.

Palabras clave: Competitividad; PYMES exportadoras; Internacionalización; Estrategia

\section{STUDY OF THE EXPORTING SMES COMPETITIVENESS IN THE VILLA MARÍA'S REGION (ARGENTINA)}

\section{ABSTRACT}

This paper addresses the problematic of competitiveness of exporting SMES in the region of Villa Maria (Argentina). A comparison with its Iberoamerican peers is undertaken by 


\section{NICOLÁS SALVADOR BELTRAMINO" MARIA CECILIA CONCI** JUAN MARCELO INGARAMO*** LILIA CARINA GAZZANIGA****}

using the methodology provided by FAEDPYME (Foundation for strategic analysis and development of small and medium enterprises), in order to make comparable the research results. The purpose is to determine competitiveness of our companies compared with the rest of Iberoamerica.

Keywords: Competitiveness; Exporting SMES; Internationalization Strategy.

\section{INTRODUCCIÓN}

Las micro, pequeñas y medianas empresas (PYMES) han sido fuente de numerosos estudios a lo largo de los últimos años, debido principalmente a su gran capacidad para la creación y mantenimiento de los niveles de empleo, así como al papel primordial como dinamizadoras de las economías de los diferentes países, contribuyendo fuertemente a la generación de riqueza, así como de manera importante en el PIB nacional. Esto ha permitido un mayor conocimiento sobre sus características y sus relaciones con el entorno económico. No obstante, Ias PYMES siguen necesitadas de fundamentos operativos que, de forma continua, pongan de manifiesto su problemática y sus estrategias al objeto de facilitar la toma de decisiones, tanto desde un punto de vista de política interna de la empresa para su gestión, como de política nacional o internacional, para determinar y fijar programas de actuación acertados y con suficiente tiempo de anticipación.

En este trabajo, nos vamos a ocupar de determinar las características de un tipo particular de estas PYMES, que son las que dedican parte de sus operaciones al desarrollo de negocios a nivel internacional, es decir, las consideradas como exportadoras.

El objetivo de este trabajo es analizar la situación de las PYMES exportadoras de la región de Villa María, Argentina, considerando los principales factores competitivos.

\section{METODOLOGÍA}

Para llevar a cabo este trabajo, se realizó un estudio empírico a partir de la información proveniente de cuestionarios a PYMES exportadoras locales, dirigidos al gerente de la empresa dentro de un radio de $100 \mathrm{~km}$ de la ciudad de Villa María, incluyendo en la muestra empre-

\footnotetext{
* Magíster, investigador Universidad Nacional de Villa María, Argentina. Correo-e: nico_beltra@hotmail.com.

* Especialista, investigador Universidad Nacional de Villa María, Argentina. Correo-e: vicerrectorado@unvm.edu.ar.

*** Especialista, investigador Universidad Nacional de Villa María, Argentina. Correo-e: iscecontabilidad@hotmail.com

**** Contadora pública, investigadora Universidad Nacional de Villa María, Argentina. Correo-e: carinagazzaniga@hotmail.com.

Recibido: 9 de noviembre de 2015, aceptado: 1 de abril de 2016.

Para citar el artículo: Beltramino, N.S.; Conci, M.C.; Ingaramo, J.M.; Gazzaniga, L.C. (2016). "Estudio de competitividad de las PYMES exportadoras de la región de Villa María, Argentina", en Sotavento MBA, n. ${ }^{\circ} 27$, pp. 122-131. D0I: http:// dx.doi.org/10.18601/01233734.n27.09
} 
sas que cuentan con entre 5 y 250 trabajadores. En esto se sigue la guía establecida por Ia FAEDPYME, a los efectos de poder obtener conclusiones comparativas.

\section{FUNDAMENTO TEÓRICO}

El presente marco teórico ha sido adaptado y sintetizado de los informes elaborados por la FAEDPYME para el análisis de la competitividad de las PYMES iberoamericanas.

Desde esta perspectiva, la clave del éxito competitivo se sitúa en elementos pertenecientes a la propia empresa, una idea que conceptualmente ha propiciado el desarrollo de la Teoría de Recursos y Capacidades (Grant, 1991).

Esta perspectiva, frente a otras, sugiere que las diferencias existentes en los niveles de éxito alcanzados por las empresas se explican, más que por las características estructurales del sector en el que actúa, por las diferentes dotaciones de recursos y capacidades de las organizaciones (Barney, 1991).

Un primer acercamiento a los factores competitivos permite clasificarlos en dos grandes grupos: externos e internos.

Entre los primeros, se sitúan aquellas variables que se derivan del entorno de actuación de la empresa. Nos referimos, por un lado, a las variables sociales, económicas, políticas y legales que afectan por igual al funcionamiento de las empresas situadas en un determinado lugar y, por otro, al conjunto de características estructurales que moldean el entorno sectorial o industrial bajo el cual se desarrolla la competencia entre empresas.

Variables como el número de competidores en el mercado y su distribución por cuotas, el grado de integración vertical existente, el número de compradores y su distribución, el grado de intensidad de las barreras de entrada y salida del sector, el nivel de diferenciación del producto, la existencia de productos sustitutivos, el nivel de información de cada empresa, entre otras, son los pilares en los que se apoya este efecto.

Los factores internos se refieren a las variables propias de cada empresa. Nos referimos a los recursos físicos, técnicos, financieros, humanos y a las habilidades y conocimientos tecnológicos, organizativos y directivos.

Aquellos recursos o habilidades que muestren un mayor potencial competitivo serán considerados como críticos o estratégicos y las empresas deberán realizar las inversiones necesarias para mantenerlos y desarrollarlos en el medio y largo plazo.

Los recursos por sí solos no generan renta alguna; es necesaria su adecuada coordinación para conseguir ventajas sobre los competidores (Grant, 1991; Amit y Schoemaker, 1993). Las capacidades son precisamente esto, la facultad de gestionar adecuadamente los recursos para realizar una determinada tarea dentro de la empresa (Grant, 1991).

El conocimiento de los recursos estratégicos contribuirá a profundizar en el conocimiento de las PYMES, al mismo tiempo que será de utilidad para la gestión práctica de este tipo de empresas. Para que las PYMEs exportadoras mejoren su posición relativa respecto a su competencia, es necesario que los directivos sean capaces de identificar las áreas clave de gestión, es decir, aquellos elementos que la empresa debe asegurar para ser competitiva (Pil y Holwelg, 2003). Concretamente, se analiza el papel jugado por la cooperación entre empresas, los recursos humanos, la innovación y la tecnología, la calidad, las tecnologías de la información y comunicación, y los recursos financieros, tal como se puede observar en el siguiente gráfico. 
Gráfico 1. Áreas clave de gestión
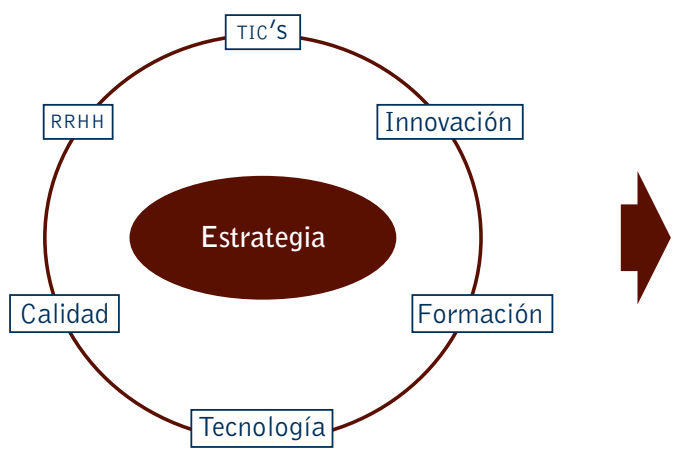

Planificación,

financiación

y gestión

Rendimiento

Fuente: Elaboración propia.

\section{RESULTADOS}

\section{CARACTERÍSTICAS DE LAS EMPRESAS}

Para comenzar, las empresas exportadoras analizadas desarrollan actividades industriales en más de un 70\%, actuando en el sector agroalimentario poco más del $40 \%$. Esto, sumado a la metalmecánica, que en su mayoría se dedica a la elaboración de herramientas para el agro, arroja que el $60 \%$ de la actividad económica exportadora de la región proviene del sector agroindustrial, demostrando el potencial de región en esta materia.

Gráfico 2. Sector de actividad

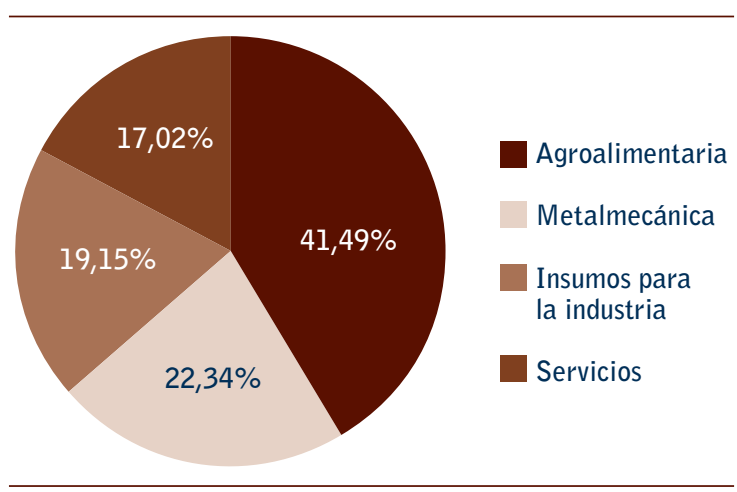

Fuente: Elaboración propia.

En lo referente al tamaño de las empresas, el $80 \%$ posee más de 10 trabajadores, lo que es explicable debido a los volúmenes necesarios para llevar adelante la actividad exportadora.

Gráfico 3. Tamaño de las empresas

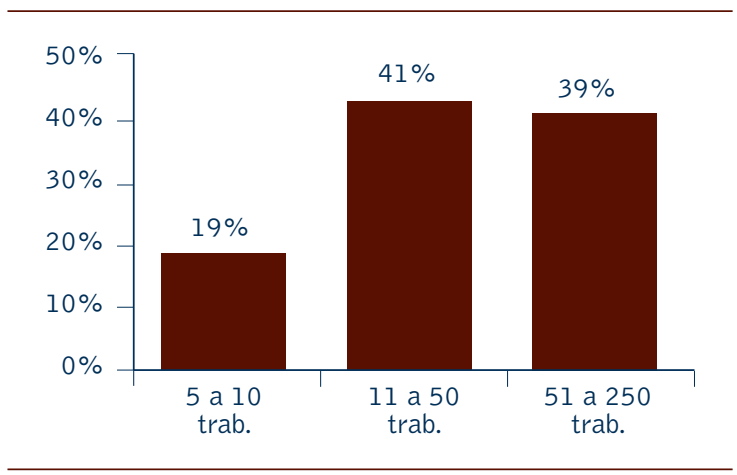

Fuente: Elaboración propia.

Gráfico 4. Edad de la empresa y el gerente

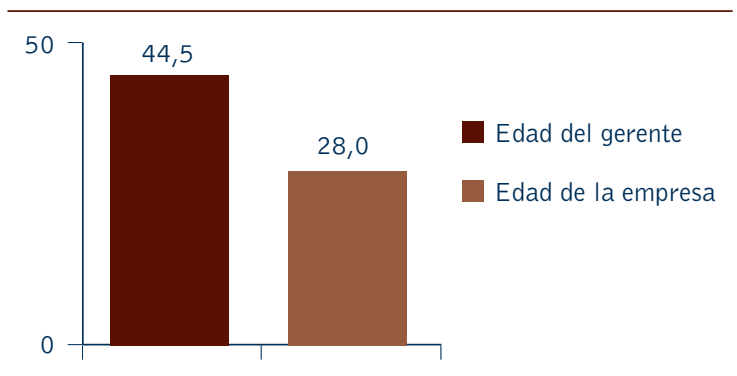

Fuente: Elaboración propia.

Al analizar la edad de las empresas de la muestra, nos encontramos que el promedio de antigüedad de las consultadas es de 28 años, con un mínimo de 3 años de vida y un máximo 
de 116 , siendo el valor modal de 10 años. En lo que se refiere a la edad de la persona a cargo de la dirección general de la empresa, se encuentra en los 44 años y medio, siendo el responsable de menor edad de 28 años, y el mayor, de 80 años, con un valor modal de 50 años.

Gráfico 5. Tendencia sobre empleados

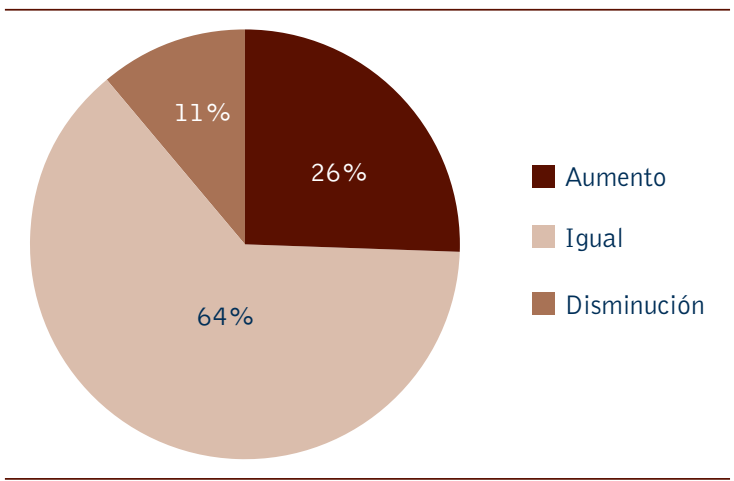

Fuente: Elaboración propia.

Gráfico 6. Tendencia sobre ventas

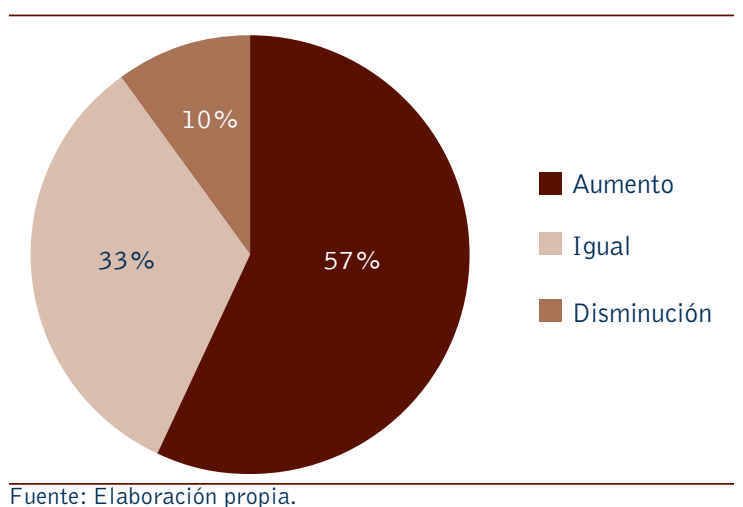

Gráfico 7. Tendencia clima empresarial

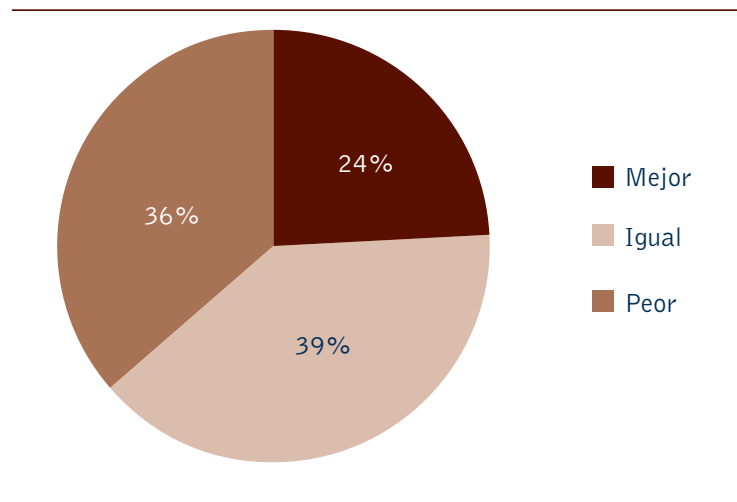

Fuente: Elaboración propia.
Con relación a las perspectivas futuras, en lo referido a la demanda de nuevos empleados, un alto porcentaje, más del $60 \%$, estima que las perspectivas se van a mantener en los niveles actuales de ocupación. Un $26 \%$ de los encuestados estima que se producirá un aumento en la demanda, mientras que solamente un $11 \%$ prevé una disminución.

Las tendencias en cuanto a la evolución de las ventas proyectadas un $57 \%$ estiman que se producirá un incremento, en tanto que solo el $10 \%$ estima una baja en el volumen de operaciones, y un tercio de los entrevistados estima que los volúmenes de operaciones se mantendrán.

\section{ANÁLISIS DE EXPORTACIONES}

Las empresas consultadas tienen como destino principal de sus exportaciones al mercado de Brasil, con un $46,15 \%$, seguidos del resto de los países latinoamericanos, que totalizan un $64 \%$. El tercer destino es el mercado europeo, con una participación del $47 \%$, y luego, con porcentajes de entre el 25 y el $30 \%$, se ubican África, Medio Oriente, Lejano Oriente y Estados Unidos.

Gráfico 8. Exportaciones por mercados

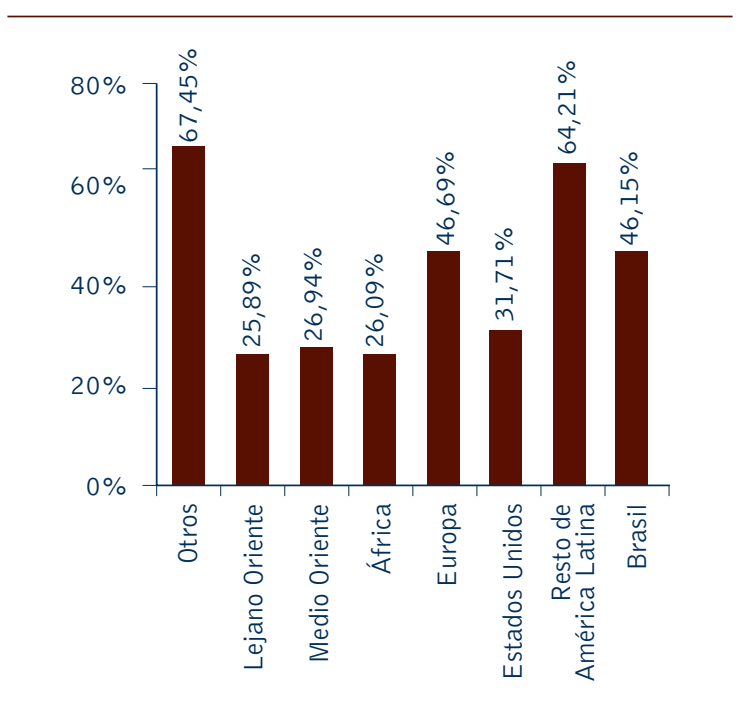

Fuente: Elaboración propia. 


\section{ANÁLISIS ESTRATÉGICO}

Comenzando con el estudio de este elemento competitivo, la estrategia empresarial, observamos que las empresas consultadas manifiestan que la principal amenaza, entre las fuerzas competitivas, es la rivalidad de la competencia en el sector en el que actúan, con una ponderación del $70 \%$, seguidos por el poder de negociación de los clientes y proveedores, con poco más del $50 \%$ y, en último término, con una ponderación del $45 \%$, le siguen las barreras de ingresos al sector y la posibilidad de creación de productos sustitutos.

Gráfico 9. Fuerzas competitivas

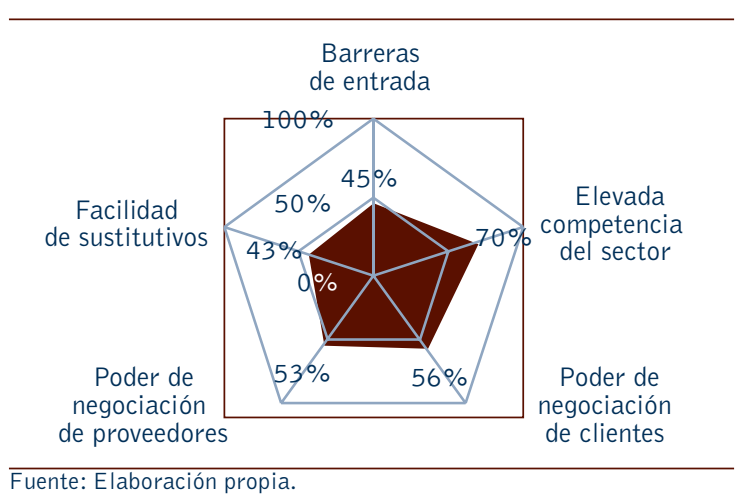

En cuanto a la realización de planes estratégicos, un alto porcentaje, prácticamente el $70 \%$, manifestó que realizan planes y, de ellos, los realizan casi el $70 \%$ también a menos de un año de plazo. Lo que implica que prácticamente la mitad de las empresas analizadas realiza planificaciones de hasta un año, y que menos del $20 \%$ lo hace con períodos mayores al año.

Gráfico 10. Exportaciones por mercados

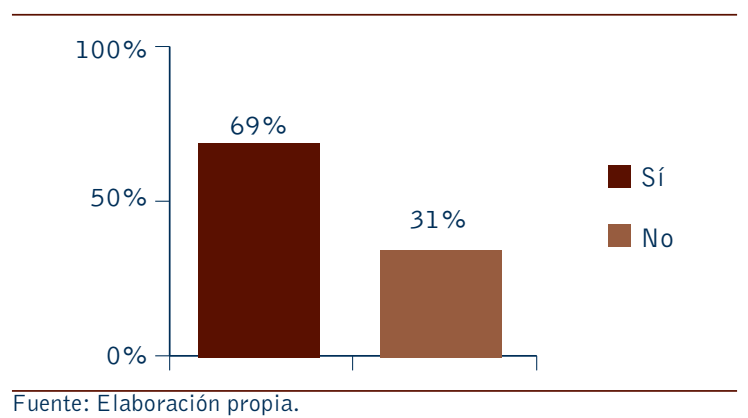

Gráfico 11. Manejo de los cambios

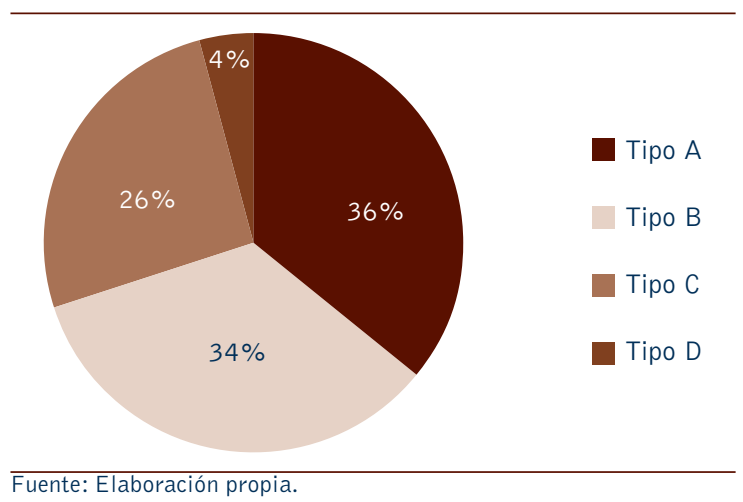

Consultadas sobre qué estrategia utilizan frente a los cambios o procesos de mejoras, poco más de un tercio suele realizar cambios y mejoras en los productos/servicios y mercados con relativa frecuencia, primando el desarrollo de productos, aun con el riesgo de que estas innovaciones no tengan éxito. En tanto, otro tercio prefiere mantener una base relativamente estable de productos/servicios y mercados, mientras que al mismo tiempo desarrolla de forma selectiva nuevos productos/servicios y mercados, tratando de imitar a las empresas que ya los desarrollaron y tuvieron éxito. Casi una cuarta parte de las empresas analizadas prefieren ofrecer un conjunto relativamente estable de productos/servicios para un mercado relativamente estable. No están interesadas en las modificaciones, sino que se concentran en la mejora continua del trabajo dentro de su campo de actuación. Solamente un escaso número de empresas, un $4 \%$ de las analizadas, reconocen que no cuentan con un área de producto-mercado duradera y estable. Normalmente, actúan forzadas por las presiones del entorno y de la competencia.

\section{ESTRUCTURA ORGANIZATIVA DE LA EMPRESA}

Al analizar la conformación funcional de las empresas bajo estudio, pudimos observar que, 
en su gran mayoría, prácticamente el 90\%, cuentan con funciones referidas a la administración o contabilidad, así como comercialización y ventas y la función de producción, y compras con casi el $80 \%$. Si nos referimos a funciones relativas a la gestión de la calidad, observamos que en las tres terceras partes de la muestra se encuentra presente esta actividad, siendo las cuestiones relativas a la gestión de los recursos humanos y la investigación y desarrollo las que se hallan presentes solo en la mitad de los casos estudiados.

Gráfico 12. Estructura de la empresa

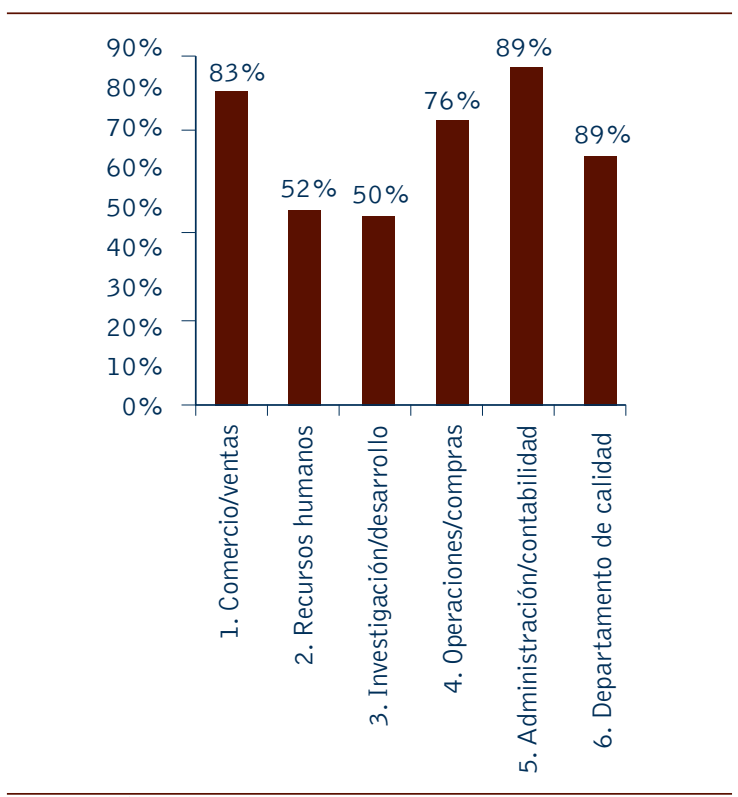

Fuente: Elaboración propia.

\section{PROCESOS DE CAMBIOSY MEJORAS}

Consultadas sobre el grado de cambio y mejoras efectuadas por la empresa en cuanto a productos/servicios, nos respondieron en más de un $80 \%$ de manera afirmativa que habían desarrollado cambios o mejoras en los produc- tos y servicios, y en casi dos terceras partes lo habían hecho sobre los sistemas de comercialización respectivos.

Gráfico 13. Productos / servicios

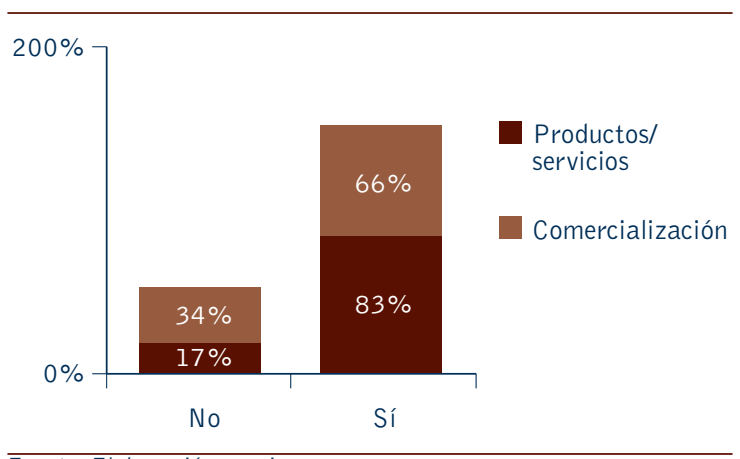

Fuente: Elaboración propia.

Gráfico 14. Procesos

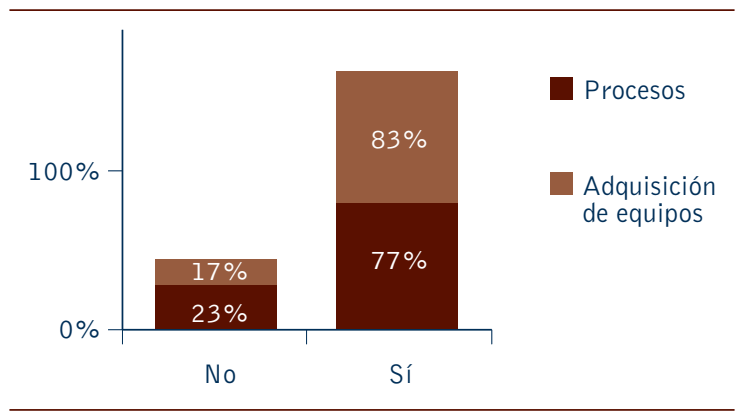

Fuente: Elaboración propia.

En cuanto a los cambios producidos en los procesos, alrededor del $80 \%$ de las empresas efectuaron cambios, tanto en los procesos de producción como de prestación de servicios, y llevaron adelante adquisición de nuevos bienes y equipos.

En última instancia, en cuanto al estudio de los cambios, analizamos los realizados en los sistemas de gestión, observando que más de dos terceras partes de las empresas consultadas respondieron que habían llevado adelante cambios en los sistemas de gestión comercial, compras y abastecimiento y en los sistemas de dirección. 
Gráfico 15. Sistemas de gestión

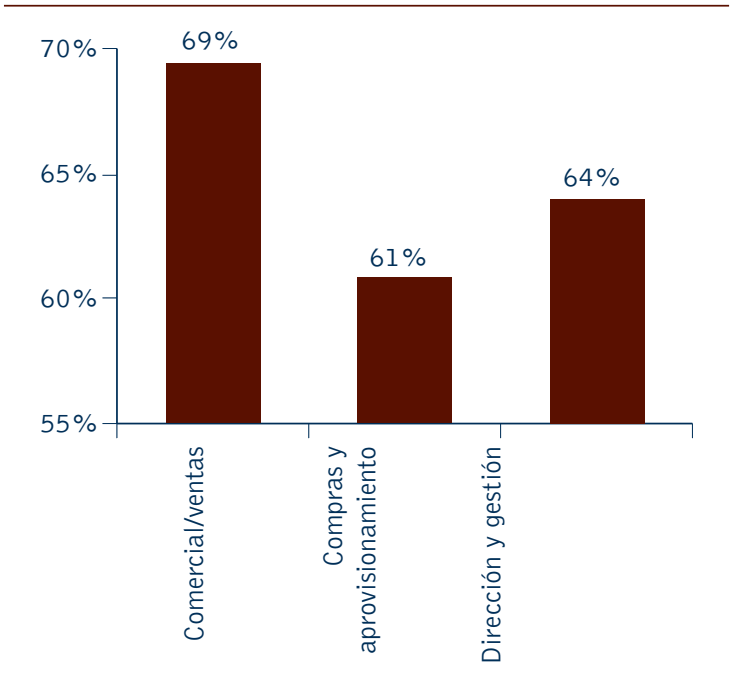

Fuente: Elaboración propia.

\section{TECNOLOGÍAS DE LA INFORMACIÓN Y LA COMUNICACIÓN (TIC)}

Observando la gráfica sobre el manejo de la infraestructura tecnológica, podemos observar que prácticamente la totalidad de las empresas hace uso de las herramientas de Tic básicas, como lo son el correo electrónico, una página web de la empresa, realizar operaciones por esta vía y efectuar operaciones por home banking. Los aspectos tecnológicos un poco más desatendidos se refieren a la existencia de redes de información internas (intranet) y la realización de acciones de marketing virtual, siendo este un aspecto lógico, dado el escaso desarrollo que tiene hoy en la región.

Gráfico 16. Infraestructura

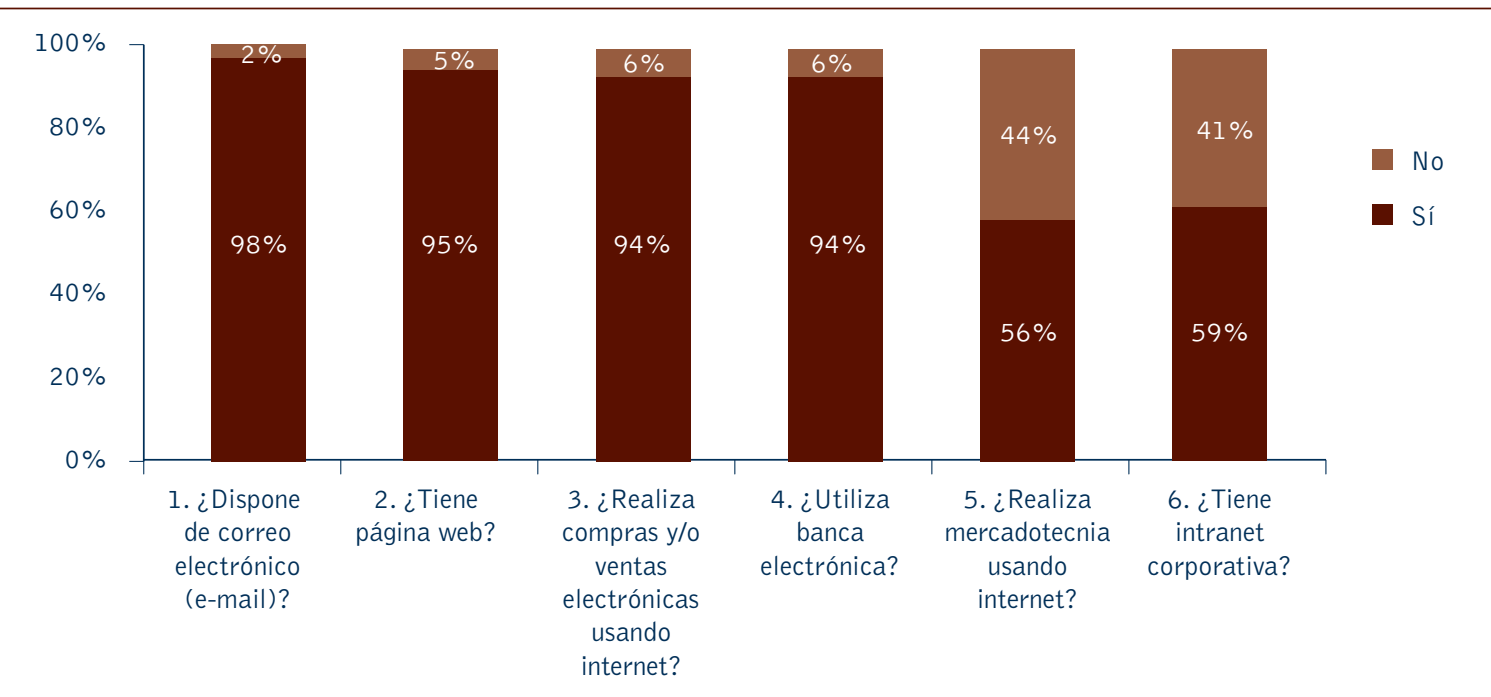

Fuente: Elaboración propia.

\section{ANÁLISIS DE COMPETITIVIDAD}

Al consultarles a las empresas sobre cómo se observan con respecto a sus competidores, casi un $60 \%$ respondió que poseen una rentabilidad superior a la de sus competidores más próximos, y más de la mitad manifiesta que ofrecen productos de mejor calidad, disponen procesos más eficientes, cuentan con clientes más satisfechos, tienen mayor adaptación a los cambios que se producen en los mercados y crecen más que sus competidores inmediatos. 


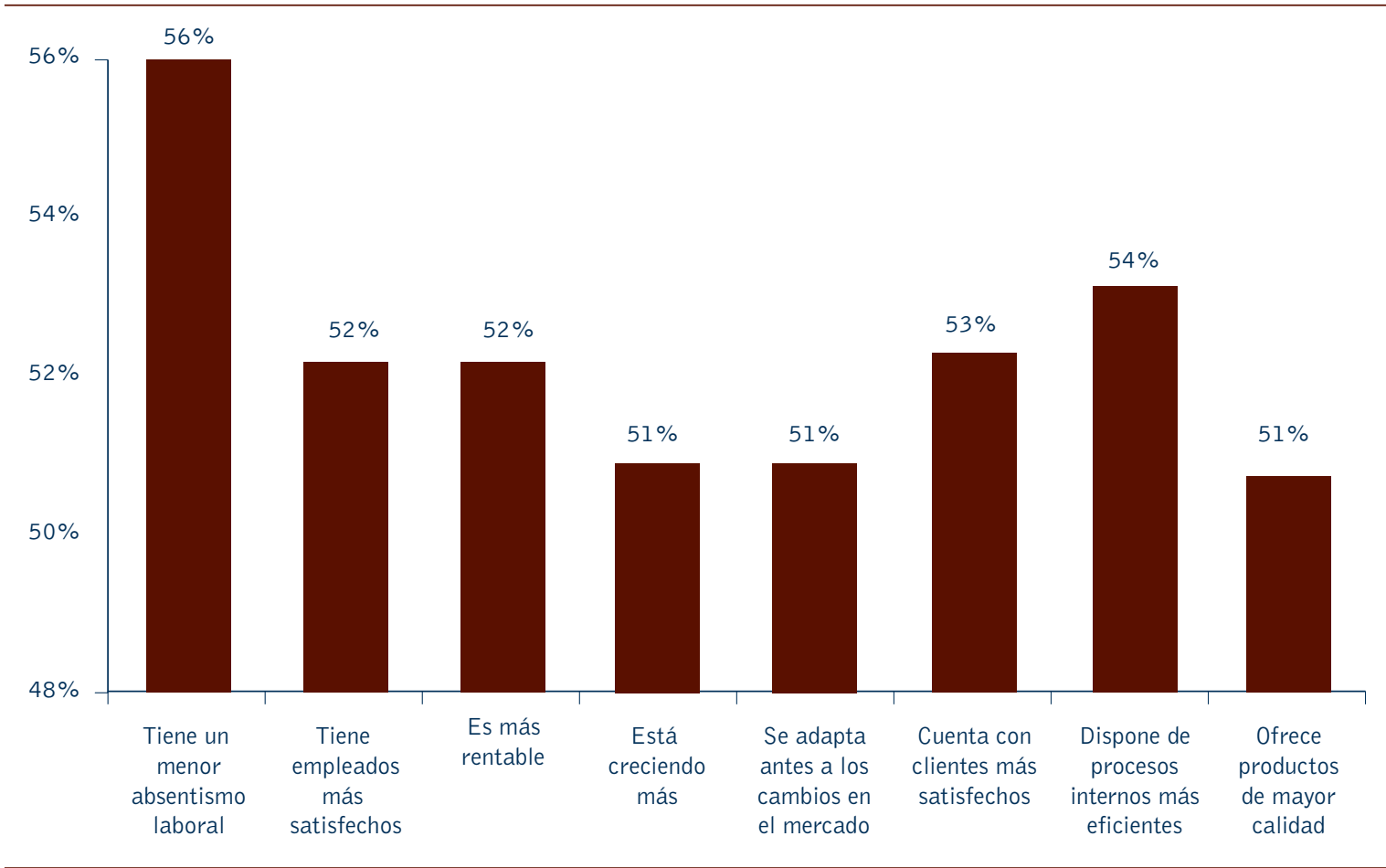

Fuente: Elaboración propia.

\section{CERTIFICACIONES DE SISTEMAS DE CALIDAD}

Poco más del $40 \%$ de las empresas manifiesta poseer sistemas de certificación de la calidad; una cuarta parte, si bien no los poseen aún, sí se encuentran en proceso de certificación, mientras que casi el tercio restante no lo consideran importante como factor competitivo.

\section{Gráfico 18. Dispone de normas de calidad}

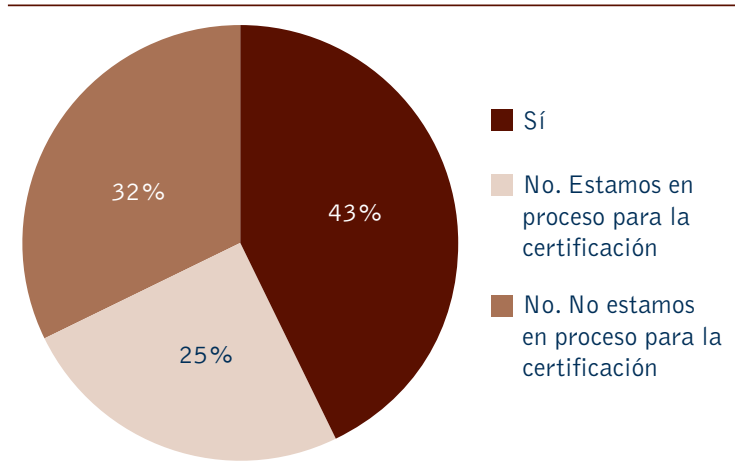

Fuente: Elaboración propia.
Factores de éxito de las PYMES exportadoras

Las empresas que tienen una posición competitiva mejor que sus competidores se caracterizan por:

- Ser empresas medianas.

- Estar en el segmento de las más jóvenes.

- Son no familiares.

- Tienen un gerente con estudios universitarios.

- Realizan plan estratégico formal.

- Tienen mayores porcentajes de acuerdos de cooperación con otras empresas, especialmente en las áreas de logística y de I+D.

- Siguen una estrategia exploradora.

- Tienen más diferenciadas en sus estructuras organizativas las áreas de I+D, Recursos Humanos y Calidad.

- Aplican de forma significativa, por encima del resto, todos los procesos de 
recursos humanos, destacando los de prevención de riesgos laborales, administración de personal, evaluación del desempeño, reclutamiento y selección, y formación.

- Tienen una posición tecnológica fuerte.

- Son empresas con certificación de calidad o que están en proceso de certificación.

- Cuentan con un mayor número de áreas de innovación.

- Hacen un uso avanzado de las Tecnologías de la Información y Comunicación (TIC).

- Tienen implantada la contabilidad de costos.

- Utilizan presupuestos de ingresos y gastos anuales.

- Hacen un mayor análisis de la situación económica y financiera.

\section{CONCLUSIÓN}

Por lo analizado en todo el estudio, que abordó diferentes aspectos de las PYMES exportadoras situadas en Villa María y la región, y comparado con el estudio de FAEDPYMES realizado en 20 países iberoamericanos, los resultados arrojados por ambos estudios son muy similares a nivel general, con la diferencia de que en el estudio realizado en las PYMEs exportadoras de Villa María y la región se observan algunos puntos con ventajas con respecto a la generalidad de las empresas iberoamericanas, posiblemente por el tipo de actividad desarrollada, ya que requiere mayores niveles de exigencias que las que se dedican al comercio doméstico.

\section{REFERENCIAS}

Álvarez, J. C.y García, E. (1996). "Factores de éxito y riesgo en la PYME: diseño e implantación de un modelo para la mejora de la competitividad", en Economía Industrial, n. ${ }^{\circ} 310$, pp. 149-161.

Amit, R.; Schoemaker, P. J. (1993). Strategic Assets and Organizational Rent. Strategic Management Journal, vol. 14, pp. 33-46.

Barney, J. B. (1991). "Firm Resources and Sustained Competitive Advantage", en Journal of Management, vol. 17, n. ${ }^{\circ}$ 1, pp. 99-120.

García Pérez de Lema, D.; Marín Hernández, S. y Martínez García, F.J. (2006). "Contabilidad de costos y rentabilidad en la Pyme", en Contaduría y Administración (Revista publicada por la Facultad de Contaduría y Administración de la UNAM - México), vol. 218, enero abril, pp. 39-59.

Grant, R. M. (1991): "The Resource-Based Theory of Competitive Advantage: Implications for Strategy Formulation", en California Management Review, Spring, vol. 33, n. ${ }^{\circ}$ 3, pp. 114-135. 